\title{
Bundesstatistikgesetz 2000 - einige Anmerkungen aus supranationaler Perspektive
}

\author{
Christian Engelage \\ Adviser on Statistical Legal Matters and Confidentiality \\ Luxemburg
}

\begin{abstract}
Zusammenfassung: Das neue Bundesstatistikgesetz 2000 überläßt der Praxis einen großen Freiraum, wie die Spielregeln für die Grundprinzipien der amtlichen Statistik in Österreich tatsächliche Geltung erlangen. Dies gilt sowohl für das Verhältnis zwischen Regierungsinformation einerseits und dem Anspruch der Allgemeinheit auf Teilhabe an und Kontrolle durch öffentliche Information, als auch für die Relevanz der Prinzipien für alle an der Statistikproduktion beteiligten Stellen und insbesondere für den Raum, in dem technical autonomy praktiziert wird. Hier wird ein durch entsprechende Auslegung bzw. durch gesetzlich nachrangige Maßnahmen nutzbarer Anpassungspielraum gesehen.
\end{abstract}

Praktische Probleme müssen in Bezug auf die Definition personenbezogener Daten und den Blickwinkel bei der Bestimmung der Identifizierbarkeit gelöst werden, insbesondere für die Frage wissenschaftlicher Nutzung von Statistikdaten. Hier wird ein Anpassungsbedarf an europäische Normenstandards gesehen, ebenso wie bei dem immer noch weitgehend bestehenden Gesetzesvorbehalt bei der Nutzung von Verwaltungsdaten und bei der Aufbereitung von Statistiken, die auf verschiedenen Rechtsgrundlagen erhoben wurden.

\section{Einführung}

Das neue österreichische Bundesstatistikgesetz 2000 hat im Vorfeld seiner Anwendungspraxis bereits viel Lob erfahren. Das ist verständlich, zeichnet sich das Regelungswerk doch durch einen erfreulich klaren Aufbau und die Liebe zum Detail aus, was dem Laien die äußerst verzwickten Mechanismen der Organisation, der Entscheidungsabläufe und der Durchführung amtlicher Statistiken verständlich erscheinen läßt. Für dieses Bemühen gebührt den Autoren hohe Anerkennung, denn Transparenz ist eines der wichtigen Prinzipien, ohne die die amtliche Statistik vergeblich um Akzeptanz werben müßte. Im Folgenden wird bewußt von einer Würdigung des Gesamtwerkes abgesehen. Statt dessen wird nur auf die europäische Dimension anhand einiger Beispiele eingegangen, die besondere Aufmerksamkeit für die Praxis verdienen. Hier wurde offenbar der Spielraum genutzt, der bei der Implementierung der entsprechenden Gemeinschaftsrechtsakte gesehen wurde (vgl. Erläuterungen A. Allgemeiner Teil).

Maßstab für die Kommentierung sind die FUNDAMENTAL PRINCIPLES OF OFFICIAL STATISTICS (im folgenden „Grundprinzipien“), die von der Statistischen Kommission der Vereinten Nationen am 14. April 1994 verabschiedet wurden und die nach ihrer Präambel für alle Staaten gültig sein sollen und universelle Bedeutung haben. Diese Prinzipien sind inhaltlich in die VERORDNUNG (EG) Nr. 322/97 DES RATES vom 17. Februar 1997 über die Gemeinschaftsstatistiken (Abl. Nr. 52 vom 22.2.1997, S. 1) 
(im folgenden „Verordnung über Gemeinschaftsstatistiken“) übertragen worden und unterliegen auch den Empfehlungen des Europarats Nr. R (97) 18 vom 30. September 1997 über The protection of personal data collected and processed for statistical purposes (im folgenden ,Empfehlungen des Europarates“). Die genannten Akte sind u.a. auch von zentraler Bedeutung für die Kompatibilität der nationalen statistischen Systeme mit dem acquis communautaire in den Verhandlungen mit den Beitrittskandidaten zur EU.

\section{Amtliche Statistik - unverzichtbares Element einer de- mokratischen Gesellschaft}

Das erste Grundprinzip lautet wie folgt: „Official statistics provide an indispensable element in the information system of a democratic society, serving the government, the economy and the public with data ... To this end, official statistics ... are to be compiled and made available on an impartial basis ... to honour citizens' entitlement to public information."

An diesem Grundsatz ist die Formulierung des $\S 1$ ausgerichtet, der die Bundesstatistik in der Zielbestimmung als ein Informationssystem des Bundes bezeichnet.

Damit wird die Statistik nicht, wie in den Statistikgesetzen bisher üblich, isoliert als Sichtbarmachung von Massenphänomenen beschrieben. Sie wird vielmehr als ein Baustein in den Gesamtkontext öffentlicher Informationsinfrastruktur integriert. Diese zutreffende Beschreibung, die noch um einen Hinweis auf Qualität und Repräsentativität ergänzt werden könnte, gibt der Statistik nicht nur eine führende Rolle beim staatlichen Informationsmanagement, sondern rechtfertigt auch einen koordinierenden Einfluß auf andere staatliche Informationsquellen, wie bei der Einrichtung und Änderung von öffentlichen Registern, bei der nach $\S 10$ Abs. 4 auf die Erfordernisse der Bundesanstalt „Statistik Österreich“ - noch treffender wäre der Verweis auf die „Bundesstatistik“ gewesen - Bedacht zu nehmen ist. Dazu gehört auch das Wissen um die Existenz vorhandener Informationsquellen in der Verwaltung, das für die Statistikanstalt mit $\S 10$ Abs. 5 erschlossen wird.

Demgegenüber wird neben den im Gesetzestext verankerten Regierungsfunktionen die gleichbedeutende Stellung des ,demokratischen“Elements nicht so deutlich sichtbar.

Es mag Zufall sein, daß nach dem Wortlaut des $\S 1$ die Entscheidungsvorbereitung und Kontrolle ihrer Maßnahmen durch Statistik lediglich den Regierungsorganen zu dienen bestimmt ist und Wissenschaft, Wirtschaft und Öffentlichkeit diesbezüglich nicht direkt angesprochen werden. Doch auch im materiellen Bereich des Gesetzes findet man zuhauf Regelungen, die die Ausrichtung der Statistik auf Serviceleistungen für die Regierungsstellen absichern, ohne daß die korrespondierende Rolle als öffentliches Gut vergleichsweise sichtbar bleibt.

So entspricht es sicherlich der notwendigen Rücksicht auf die budgetären Restriktionen und kann auch eine datenschutzrechtliche Komponente haben, wenn z.B. die Anordnung von Statistiken durch Rechtsverordnung darauf zu beschränken ist, was für die Wahrnehmung von Bundesaufgaben benötigt wird und ein angemessenes Verhältnis zur Bedeutung der Bundesaufgabe bestehen muß. Und Initiator von Verordnungsstatistiken ist der zuständige Bundesminister. Wie ist gewährleistet, daß dessen Sicht der Notwen- 
digkeit, für die er die politische Verantwortung trägt, auch das Informationsinteresse der Allgemeinheit an der Transparenz und Kontrollierbarkeit seiner eigenen politischen Entscheidungen berücksichtigt? Hier könnte man sich vorstellen, daß z.B. einer der eingerichteten Räte diese Funktion tragen könnte. Nun obliegt dem Statistikrat zwar die Abgabe von Stellungnahmen zu Verordnungsentwürfen ( 97 Abs. 1 Z 3), er setzt sich jedoch ausschließlich aus Vertretern von Regierung und anderen öffentlichen Einrichtungen zusammen. Anders ist es bei der Statistischen Zentralkommission und den Fachbeiräten nach $\S 63$. Diesen gehören - neben den Vertretern wie bei $\S 47$ - die erforderliche Anzahl von im Berufsleben stehenden Fachleuten des wirtschaftlichen, wissenschaftlichen und kulturellen Lebens an. Aus dem Wortlaut geht nicht hervor, ob mit dieser Auflistung die sozial relevanten Gruppierungen gemeint sind und ob die Erforderlichkeit der Anzahl daran gemessen wird, daß diese in der Zentralkommission bzw. im Fachbeirat ggf. ein Gegengewicht zu den amtlichen Vertretern darstellen können. Und bezüglich der Aufgaben: die Zentralkommission berät in Fragen von allgemeiner Bedeutung; für einzelne Fachgebiete obliegt diese Aufgabe den Fachbeiräten. Umfassen diese allgemeinen Fragen auch generell die Abgabe von Stellungnahmen zu Verordnungsentwürfen, wie sie in der Kompetenz des Statistikrates liegen? Man wird die Vorschriften insoweit nicht kleinlich auslegen (dürfen).

Teil des demokratischen Elementes ist nicht nur die Gestaltung des Programms der Statistik, sondern auch die Verfügbarkeit der Ergebnisse. Nach dem Grundsatz in $\S 19$ sind die Statistiken einschließlich der Metadaten unverzüglich der Öffentlichkeit zugänglich zu machen. Das entspricht voll den internationalen Standards. Es fragt sich jedoch, was sich hinter der Möglichkeit verbirgt, die Veröffentlichung durch Rechtsakte einschränken zu können. Amtliche Statistiken sind zur Veröffentlichung bestimmt, sie haben in der Regel auch eine Rechtsgrundlage, die die Informationsinhalte legitimiert. Statistische Rechtsakte der EG, auf die sich der Verweis auf $\S 4 \mathrm{Z} 1$ bezieht, enthalten keine Veröffentlichungsverbote. Bei den genannten Staatsverträgen mag eine regierungsamtliche Vertraulichkeit vereinbart werden, dann handelt es sich aber nicht um amtliche Statistiken. Ebensowenig sind solche Themen relevant für das Programm amtlicher Statistiken, deren Vertraulichkeit aus Gründen der Staatssicherheit geboten sein könnte.

Mißverständnissen über die Rolle amtlicher Statistik könnte durch entsprechenden Erläuterungen vorgebeugt werden. Diese könnten auch darauf hin wirken, daß $\S 30$ Abs. 3 so anzuwenden ist, daß der zuständige Minister unverzüglich, und die Öffentlichkeit zeitgleich über die Ergebnisse der Statistiken informiert werden. Wenn aus anderen Gründen in $\S 30$ Abs. 3 unterschiedliche Formulierungen bezüglich der zeitlichen Abfolge der Unterrichtung von Ministerien und Öffentlichkeit gewählt sein sollten, dann würden diese erst recht verdienen, transparent gemacht zu werden.

\section{Relevanz der Prinzipien für die gesamte amtliche Sta- tistik}

$\S 14$ listet in enger Anlehnung an die Formulierungen in der VO über Gemeinschaftsstatistiken und Artikel 285 des EG-Vertrages die Prinzipien auf, denen die Organe der Bundesstatistik bei der Erfüllung ihrer Aufgaben zu folgen haben. Das „Organ“ Bundesanstalt 
hat daneben noch die Grundsätze in $\S 24$ zu beachten, die zwar z.T. andere Formulierungen als $\S 14$ verwenden, entgegen den Ausführungen im BESONDEREN TEIL aber nicht über diejenigen in der Verordnung über Gemeinschaftsstatistiken hinaus gehen.

Organe der Bundesstatistik umfassen nach $\S 3$ Z 19 neben der Bundesanstalt ,Statistik Österreich“" auch solche Dienststellen, denen durch Rechtsakt im Einzelfall die Erstellung bestimmter Statistiken übertragen worden ist. Keine Organe der Bundesstatistik sind demnach, und das entspricht durchaus den entsprechenden Regelungen der Verordnung über Gemeinschaftsstatistiken, die aufsichtführenden Ministerien bzw. das Bundeskanzleramt, die eine vergleichsweise starke Stellung in der Statistik haben, z.B. mit der Rechtssetzungskompetenz, mit dem Entsendungsrecht maßgeblicher Vertreter in die Räte und nicht zuletzt mit der Rechtsaufsicht, die die Organe der Bundesanstalt gem. $\S$ 54 Abs. 2 dazu verpflichten kann, bei abweichenden Auffassungen den der Rechtsanschauung des Bundeskanzlers und des Bundesministers entsprechenden Rechtszustand unverzüglich herzustellen. Es wird eine Aufgabe der Vertrauensgewinnung sein, transparent zu machen, daß die Regierungsstellen über den Wortlaut des Gesetzes hinaus bei der Wahrnehmung ihrer statistikrelevanten Aufgaben ebenfalls der Bindung an die Grundprinzipien unterliegen und diese folglich allgemein für die Durchführung der Bundesstatistik gelten.

\section{Technical autonomy}

Der Entscheidungsrahmen, den die Statistischen Ämter in abschließender Verantwortung üblicherweise vertreten, wird mit dem Begriff technical autonomy beschrieben. Hierbei geht es um Weisungsfreiheit innerhalb der Behördenhierarchie und um Zurückhaltung bei politischer Einflußnahme. Technical autonomy betrifft insbesondere drei Bereiche: die Wahl sachgerechter Methoden und Verfahren, die Gewährleistung der statistischen Geheimhaltung und die Sicherstellung der zeitgerechten Veröffentlichung aller relevanten statistischen Ergebnisse. Diese Belange sollen sich im Gesetzestext nachvollziehbar wiederfinden.

\subsection{Wissenschaftliche und methodische Fragen}

In allen wissenschaftlichen und methodischen Fragen ist der fachliche Leiter der Bundesanstalt weisungsfrei, $\S 38$ Abs. 1 .

Dies wirft zunächst die anstaltsinterne Frage der Autonomie bei dem Dirimierungsrecht des kaufmännischen Geschäftsführers auf, wenn nach dessen Meinung das gewählte Verfahren zu teuer erscheint. Dann entscheidet letztlich der Wirtschaftsrat, der sich überwiegend aus Regierungsvertretern zusammensetzt, mit einfacher Mehrheit. Dies muß der technical autonomy nicht entgegenstehen. Denn die „Politikferne“ in diesem Autonomiebereich könnte auch in der entsprechenden Geschäftseinteilung ( 338 Abs. 4) bzw. Geschäftsordnung (§ 48 Abs. 8) dadurch sichtbar gemacht werden, das der fachliche Leiter in diesen Fragen nicht überstimmt werden kann.

Eine vergleichbare Schutzregelung, wie sie nach $\S 38$ Abs. 1 für den fachlichen Leiter der Bundesanstalt besteht, ist für andere Dienststellen, die als Organe der Bundesstatistik 
gleichfalls Statistiken ausführen können, nicht ersichtlich. Die Autonomiefrage stellt sich daher auch außerhalb der Bundesanstalt.

Für diese Dienststellen, die von ihrer Aufgabenstellung her überwiegend Exekutivaufgaben haben dürften, gilt das Gebot der functional separation.

Im Gesetzestext befindet sich keine Aussage darüber, ob der Statistikteil getrennt von den Verwaltungsaufgaben zu führen ist, bzw. durch welche organisatorischen und technischen Maßnahmen die Trennung sicherzustellen ist.

\subsection{Statistische Geheimhaltung}

Die statistische Geheimhaltung gilt auch gegenüber den Behörden, die Dienst- oder Fachaufsicht ausüben. Dies ist ebenfalls immanenter Bestandteil des Prinzips der functional separation. Das Statistikgeheimnis muß daher bei der Durchführung der Aufsichtsrechte nach $\S 53$ Abs. 3 berücksichtigt werden. Diese Regelung ist daher so zu lesen und anzuwenden, daß die Einsicht in Unterlagen bzw. Überprüfungen vor Ort nicht solche Informationen einschließen, die der statistischen Geheimhaltung unterliegen. Dementsprechend wird man die Aufsichtsbehörden auch als ,Dritte“ im Sinne des $\S 17$ Abs. 2 ansehen können, denen der Zugang zu vertraulichen Daten grundsätzlich verwehrt ist.

\subsection{Veröffentlichungen}

Von herausragender Bedeutung für die Gewährleistung der notwendigen Politikferne ist die Sicherstellung einer einflußfreien Veröffentlichungspraxis. Die notwendige Handlungsfreiheit hierfür läßt sich weder unter den Begriff ,,wissenschaftliche und methodische Fragen" des $\S 38$ subsumieren noch unter einen an anderer Stelle im Sinn einer Schutzvorschrift geregelten Sachbereich, wie die statistische Geheimhaltung. Der Praxis bleibt daher der Nachweis überlassen, daß auch ohne ausdrückliche gesetzliche Absicherung die Veröffentlichungen frei von politischen Einflußnahmen durchgeführt werden. Es könnte die Glaubwürdigkeit erhöhen, wenn z.B. eine Bindung durch ein vorher veröffentlichtes Veröffentlichungsprogramm eingegangen würde, in dem die relevanten statistischen Ergebnisse zu festgelegten Terminen angekündigt werden.

\section{Statistische Geheimhaltung und Datenschutz}

Einer der zentralen Regelungsgegenstände des Gesetzes ist die Verschärfung der Regelungen über das Statistikgeheimnis (Vorblatt, Inhalt Z 7). Entsprechende Restriktionen sind zumindest in Bezug auf die Definition vertraulicher Daten und die Frage des Zugangs der Wissenschaft zu diesen Informationen sichtbar. Die Gesetzesmaterialien geben keinen Aufschluß über die Motivation zu diesen Überlegungen, außer vielleicht dem Hinweis auf Anpassungsnotwendigkeiten an EU-Rechtsnormen, wie die Datenschutzrichtlinie und die Verordnung über Gemeinschaftsstatistiken. In beiden Fällen sind die gefundenen Lösungen jedoch nicht mit europäischen Maßstäben begründbar. 


\subsection{Definition ,personenbezogener Daten““}

Schutzsubjekt personenbezogener Daten nach der Definition des $\S 15$ ist der Betroffe$n e$, dessen Daten erhoben worden sind, gleich ob dieser eine natürliche oder juristische Person ist.

Diese Terminologie entspricht nicht der Definition personenbezogener Daten in der Datenschutzrichtlinie und deren Regelungsgegenstand. Dieser bezieht sich ausschließlich auf natürliche Personen. Die Datenschutzrichtlinie ist in nationales Recht umzusetzen. Das bedeutet auch, die Terminologie mit den gleichen Begriffsinhalten zu verwenden, wie sie das europäische Recht vorgibt, wenn datenschutzrelevante Fragen geregelt werden. Zwar schützt die statistische Geheimhaltung, wie es auch in $\S 15$ angelegt ist, natürliche Personen und sonstige statistische Einheiten und die Regelungsinhalte der statistischen Geheimhaltung als bereichsspezifischer Datenschutz und des allgemeinen Datenschutzes ergänzen und präzisieren sich auch, soweit natürliche Personen betroffen sind. Sie sind jedoch nicht kongruent bei den statistischen Einheiten, die keine natürlichen Personen sind. Daher wäre es angemessener, die statistikspezifische Terminologie der vertraulichen Daten zu verwenden, wie sie durch die Verordnung über Gemeinschaftsstatistiken eingeführt ist und damit auch unmittelbar in den Mitgliedstaaten gilt. Dies um so mehr, als sich bereits jetzt die europäischen mit den nationalen Erhebungsprogrammen weitgehend und noch fortschreitend überschneiden.

Die Folge ist, daß nicht nur für die Statistiken, die ausschließlich aufgrund europäischer Normen erhoben werden, das europäische Recht Anwendung findet, sondern auch für die Statistiken, für die zusätzlich eine nationale Rechtsgrundlage besteht. Diese zusätzliche - nationale Rechtsgrundlage kann ein Umsetzungsrechtsakt für eine europäische Statistikrichtlinie sein oder ein paralleler Rechtsakt zu einer entsprechenden Ratsverordnung; in beiden Fällen bleiben die Statistiken Gemeinschaftsstatistiken.

Und auch für die Statistiken, die heute noch de jure reine nationale Statistiken sind, kann den Ausführungen in den Erläuterungen zum ALLGEMEINEN TEIL nur zugestimmt werden, wonach es aus Gründen der Rechtssicherheit und der Einheitlichkeit erforderlich ist, einheitliche Datenschutzstandards und Verfahrensweisen festzulegen. Nationales Recht kann das Gemeinschaftsrecht nicht abändern. Deshalb muß die Anpassung an das Gemeinschaftsrecht erfolgen.

Den europäischen Regelungen sowohl des Datenschutzes, als auch der Gemeinschaftsstatistik entsprechend, folgt die Definition dem Ansatz der sog. faktischen Anonymisierung, wonach lediglich theoretisch bestehende und nicht ausschließbare „Restrisiken“ einer Zuordnung hinnehmbar sind. Ähnlich wie in den europäischen Rechtsakten werden als Maßstab die Mittel berücksichtigt, die vernünftigerweise bei einem Zuordnungsversuch eingesetzt werden könnten. Das bringt erhebliche Rechtssicherheit, insbesondere bei Veröffentlichungen.

\subsection{Ausschluß des Personenbezugs bei Veröffentlichungen}

Nicht übereinstimmend mit den europäischen Vorgaben ist jedoch der fiktive Blickwinkel, aus dessen Sicht die Identifizierbarkeit und die ,vernünftigerweise“ einsetzbaren Mittel zu bewerten sind. 
Während die Verordnung über Gemeinschaftsstatistiken auf die Mittel abstellt, die von einem Dritten angewendet werden könnten (Artikel 13 Abs. 1 Unterabs. 2) kommt es nach $\S 3 \mathrm{Z} 15$ darauf an, ob die betroffene Einheit für das Organ der Bundesstatistik bestimmbar ist.

Diese Unterscheidung kann zum Einen für die Veröffentlichungen und zum Anderen für den Prozeß der Erstellung der Statistiken innerhalb der Statistikdienststelle bedeutsam sein.

Die Mittel, auf die die Statistikbehörde zugreifen könnte, sind das Wissen, das aus den statistischen Erhebungen erlangt wurde, die Informationen, auf die sonst noch zugegriffen werden könnte und die technischen Verarbeitungskapazitäten. Diese Mittel sind relativ beschränkt, im Verhältnis zu dem potentiellen ,Zusatzwissen“ und der denkbaren technischen Ausstattung, die interessierten Dritten außerhalb der Statistik zur Verfügung stehen könnten. Würde man die Kontrolle der Geheimhaltung bei Veröffentlichungen auf das Reidentifikationspotential der Statistikstelle beschränken, so würde das Statistikgeheimnis eher geschwächt als verstärkt. Daher wird von einer Anwendungspraxis ausgegangen, die Veröffentlichungen auch weiterhin ausreichend sicher gegen Identifizierungen durch Dritte schützt.

\subsection{Ausschluß des Personenbezugs bei der internen Verarbeitung}

Während die Definition in der Außenwirkung tendenziell eher zu eng greifen könnte, ergibt sich für den Innenbereich das umgekehrte Bild.

$\S 15$ verlangt die unverzügliche Beseitigung des Personenbezugs, sobald dieser nicht mehr für die speziellen Tatbestände nach $\S 5$ Abs. 2 oder für eine weitere angeordnete Statistik erforderlich ist.

Beseitigung des Personenbezugs erfordert zunächst, daß die Identifikatoren entfernt werden. Die Empfehlungen des Europarates definieren diese wie folgt: „,Identifikationsdaten“ sind solche personenbezogenen Daten, die eine direkte Identifizierung des Datensubjekts ermöglichen und die für die Erhebung, Prüfung und Verknüpfung der Daten gebraucht werden, aber nicht weiter verwendet werden zur Erstellung der statistischen Ergebnisse." (freie Übersetzung aus dem Englischen). Diese, in der Terminologie des deutschen Bundesstatistkgesetzes als „Hilfsmerkmale“ bezeichneten Informationen, die lediglich der technischen Durchführung der Statistik dienen und nicht in die Ergebnisaufbereitung eingehen, umfassen Name, Anschrift und/oder eine amtlich zugeteilte und veröffentlichte Identifizierungsnummer (siehe auch Artikel 2 Nr. 7 der Verordnung Nr. 1588/90 des Rates vom 11. Juni 1990 über die Übermittlung von unter die Geheimhaltungspflicht fallenden Informationen an das Statistische Amt der Europäischen Gemeinschaften, Abl. Nr. L 151 vom 15.6.1990, S. 1).

In der Regel gewährleistet jedoch die Anonymisierung durch das Weglassen von Name und Anschrift nicht ohne weiteres die Beseitigung des Personenbezugs. Zusätzlich erforderlich ist vielfach ein weiterer Eingriff in die als Rohmaterial für die statistische Aufbereitung erhobenen Statistikdaten, der stets mit einem, z.T. erheblichen, Informationsverlust verbunden ist.

Man wird sicherlich nicht ernsthaft auf den Gedanken kommen, die Beseitigung des Personenbezugs durch Eingriff in das Aufbereitungsmaterial durchzuführen, bevor über- 
haupt eine statistische Auswertung erfolgt ist, wie immer man das mit $\S 15$ in Einklang bringt. Aber wie sieht es mit weiteren Aufbereitungen aus, die nicht zeitgleich erfolgen und die nach anderen Programmen zusammengestellt werden, z.B. die ,sonstigen“ Statistiken nach $\S 23$ Abs. 2? Diese Arbeiten erfordern die Speicherung und Verfügbarkeit der vollständigen statistischen Datensätze. Daher gilt es, in Übereinstimmung mit den Empfehlungen des Europarats, für die „Innenanomysierung“ ein Ergebnis zu finden, das die Trennung und Löschung der Identifikatoren regelt, aber die Aufbereitungsdaten verfügbar läßt. Gründe der Normenklarheit sprechen für eine erkennbare Verankerung im Gesetz.

Aufbereitung der Angaben ist übrigens auch keiner der in $\S 5$ Abs. 2 genannten Gründe, die eine Aufschiebung der Beseitigung des Personenbezugs nach $\S 15$ Abs. 1 rechtfertigen würden.

\section{4 Übermittlungen an Eurostat}

Die Bezugnahme auf personenbezogene Daten bei der Übermittlung an Dritte nach $\S 17$ Abs. 2 anstatt auf Daten, die keine direkte Identifizierung erlauben (vgl. Artikel 14 der Verordnung über Gemeinschaftsstatistiken) wirkt sich auch auf die Datenübermittlung an Eurostat aus.

Personenbezogene Daten dürfen nur übermittelt werden, wenn u.a. Rechtsakte gemäß $\S 4$ Abs. $1 \mathrm{Z} 1$ dies vorsehen. Solche Rechtsakte müssen innerstaatlich unmittelbar wirksam sein, die Anordnung statistischer Erhebungen enthalten und die Übermittlung personenbezogener Daten vorsehen.

EG-Statistiken werden durch Verordnungen oder Richtlinien angeordnet. Dabei ,gilt“ eine EG-Verordnung unmittelbar in jedem Mitgliedstaat (Artikel 249 des EG-Vertrages). Und Richtlinien, die Statistiken anordnen? Diese gelten nicht unmittelbar in den Mitgliedstaaten, sie verpflichten diese jedoch zur Anpassung der nationalen Rechtslage an die zu erreichenden Ziele. Diese Verpflichtung kann als unmittelbar wirksam unter $\S 4$ Abs. $1 \mathrm{Z} 1$ subsumiert werden.

Eine weitere Schwierigkeit, die Verordnung über Gemeinschaftsstatistiken in die Bestimmung des $\S 4$ Abs. $1 \mathrm{Z} 1$ einzuordnen, liegt darin, daß diese zwar den normativen Rahmen für die Erstellung der Gemeinschaftsstatistiken aufstellt, selbst aber keine Statistiken anordnet.

Trotzdem erscheint eine extensive Auslegung gerechtfertigt, die alle Gemeinschaftsstatistiken, gleich auf welcher konkreten Grundlage sie beruhen, in $\S 17$ Abs. 2 einbeziehen.

Denn zum Einen folgt die Gemeinschaftsstatistik einem anderen Anordnungsmechanismus als das österreichische System. Dieser beruht zunächst auf dem jeweils gültigen Mehrjahresprogramm, das den Gesamtinhalt der für diesen Zeitraum ins Auge gefaßten Statistiken umschreibt. Dieses Programm wird vom Rat in einer Entscheidung festgelegt, von der Kommission in Zusammenarbeit mit dem Ausschuß für das Statistische Programm in Jahresarbeitsprogramme aufgeschnürt und erst dann in konkrete Aktionen umgesetzt, die entweder eine die betreffende Statistik anordnende Rechtsgrundlage des Rates erhalten oder die in Absprache mit den einzelstaatlichen Stellen durchgeführt werden. Alle derartig geregelten Statistiken sind Gemeinschaftsstatistiken.

Zum anderen ist auch Artikel 14 der Verordnung über Gemeinschaftsstatistiken be- 
achtlich, wonach die Übermittlung vertraulicher Daten, die keine direkte Identifizierung erlauben, ... zwischen den einzelstaatlichen Stellen sowie zwischen den einzelstaatlichen Stellen und Eurostat zulässig ist, soweit sie für die Erstellung spezifischer Gemeinschaftsstatistiken erforderlich ist. Der gemeinschaftsrechtlich eingeräumte Erlaubnistatbestand kann nationalrechtlich nicht aufgehoben werden, so daß auch aus diesem Grund eine gemeinschaftskompatible Auslegung des $\S 17$ Abs. 2 angezeigt erscheint.

\section{Zugang der Wissenschaft zu statistischen Informatio- nen}

Gemäß $\S 31$ Abs. 1 kann der Wissenschaft Zugang zu den nicht personenbezogenen statistischen Daten gewährt werden. Den sich daraus ergebenden Umkehrschluß, daß die Verwendung von personenbezogenen Statistikdaten damit ausgeschlossen wird, regelt Abs. 3 der Vorschrift ausdrücklich und hebt dies durch Einfügen des Wortes ,auch“ noch besonders hervor.

Der gänzliche Ausschluß jeglicher Teilhabemöglichkeit der Wissenschaft an der Nutzung des öffentlich finanzierten Informationspotentials statistischer Erhebungen, das von den Statistischen Dienststellen nicht selbst erschlossen werden kann und das trotz Eliminierens der Identifikatoren noch personenbezogene Elemente enthält, erstaunt.

Diese Position war anfangs bereits von Vertretern der österreichischen Delegation in den Beratungen des Rates auf Arbeitsgruppenebene bei der Verordnung über Gemeinschaftsstatistiken vertreten worden, fand jedoch keine Unterstützung.

So bestimmt Artikel 17 Abs. 1 dieser Verordnung, daß von der Bundesanstalt „Statistik Österreich“, als der zuständigen einzelstaatlichen Stelle, Zugang für wissenschaftliche Zwecke gewährt werden kann, vorausgesetzt der Schutz der vertraulichen Daten ist ausreichend sichergestellt. Eine Kompatibilität das $\S 31$ mit dieser EG-Vorgabe läßt sich hier, anders als bei $\S 17$ Abs. 2, wegen des klaren Wortlautes in Absatz 3 nicht im Wege der Auslegung herstellen. Nach dem Grundsatz des Vorrangs des Gemeinschaftsrechts vor dem nationalen Recht ist im Zweifel der Regelung des Gemeinschaftsrechts zu folgen. Da dieses in Artikel 17 der Verordnung über Gemeinschaftsstatistiken der Wissenschaft aber weder einen Rechtsanspruch auf Zugang einräumt noch den Statistikstellen eine diesbezügliche Verpflichtung auferlegt, käme es darauf an, inwieweit die der Statistikstelle eingeräumte Entscheidungsbefugnis durch Anweisungen, auch in Form von nationalen Gesetzen, gebunden werden kann. Hier wird durchaus ein Spielraum bestehen, der aber nicht soweit gehen dürfte, daß ein Gebrauchmachen von der Befugnis gänzlich ausgeschlossen wird.

$\S 31$ erstaunt auch im Hinblick auf die allgemeinen Regeln des Datenschutzes, wie sie in der Datenschutzrichtlinie zum Ausdruck kommen.

Artikel 6 Abs. 1 Buchst. b) dieser Richtlinie enthält eine für die Wissenschaft wie auch für die Statistik gleichermaßen bedeutsame Regelung. Er fixiert den Grundsatz, daß personenbezogene Daten nur für festgelegte eindeutige Zwecke erhoben werden und nicht in einer mit diesen Zweckbestimmungen nicht zu vereinbarenden Weise weiterverarbeitet werden dürfen und stellt ausdrücklich klar: Die Weiterverarbeitung von Daten zu historischen, statistischen oder wissenschaftlichen Zwecken ist ... (lediglich in der deutschen 
Version ist hier eingefügt ,im allgemeinen“) nicht als unvereinbar mit den Zwecken der vorausgegangenen Datenerhebung anzusehen, sofern die Mitgliedstaaten geeignete Garantien vorsehen.

Bezogen auf die Daten der amtlichen Statistik bedeutet dies, daß eine Weiterverarbeitung für wissenschaftliche Zwecke - ausreichende Sicherungsmaßnahmen immer vorausgesetzt - datenschutzrechtlich unbedenklich ist. Die Verpflichtung, das nationale Recht an die Datenschutzrichtlinie anzupassen, wäre ein geeigneter Anlaß, § 31 noch mal zu überdenken.

\section{Zugang der Statistik zu Verwaltungsdaten}

Und bezogen auf die Daten des Verwaltungsbereichs bedeutet Art. 6 Abs. 1 Buchst. b) der Datenschutzrichtlinie, daß eine Weiterverarbeitung für die Zwecke der amtlichen Statistik - hier kann das Vorhandensein ausreichender Sicherungsmaßnahmen ohne weiteres unterstellt werden - ebenfalls datenschutzrechtlich unbedenklich ist. Konsequenterweise hat die Verordnung über Gemeinschaftsstatistiken in Artikel 16 Abs. 1 den entsprechenden Statistikproduzenten auch einen Zugangsanspruch auf die Datenbestände der Verwaltung eingeräumt, soweit die Daten für die Erstellung der Gemeinschaftsstatistik erforderlich sind. Dies ist auch ein wichtiger Bestandteil der Empfehlungen des Europarates (Prinzip 4.2 und 4.4 a.a.O.) und basiert auf Nr. 5 der Grundprinzipien, deren Text wie folgt lautet: Data for statistical purposes may be drawn from all types of sources, be they statistical surveys or administrative records. Statistical agencies are to choose the source with regard to quality, timeliness, costs and the burdens on respondents.

Das neue Österreichische Bundesstatistikgesetz bekennt sich zu einer vorrangigen Nutzung bereits vorhandener Daten anstelle von Befragungen. So wird z.B. in $\oint 6$ Abs. 3 bei der Anordnung der Art der statistischen Erhebung - das betrifft die zu benutzende Datenquelle - eine entsprechende Hierarchie aufgestellt. Die Art der Erhebung ist ein in der Rechtsgrundlage der Statistikanordnung festzulegender Tatbestand; fehlt die entsprechende Festlegung z.B. in einer EG-Anordnung - und das ist aus Gründen der Subsidiarität bisher regelmäßig der Fall - ist dies durch nationale Verordnung zu ergänzen ( 4 Abs. 4). Dem Zugangsrecht der Statistik entspricht die Mitwirkungspflicht der Verwaltung, d.h. in dem Umfang, in dem dies in der anordnenden Rechtsgrundlage vorgesehen ist ( $⿱ 10$ Abs. 1 und 3), wobei es für die Registerdaten nach $\S 25$ einer zusätzlichen Rechtsgrundlage nicht bedarf. Das Statistikgesetz erkennt an, daß die Statistik immer ein „,berechtigtes Interesse“ darstellt, wenn der Zugang an das Vorliegen dieser Voraussetzung geknüpft sein sollte ( $§ 6$ Abs. 4) und auch, daß gesetzlich festgelegte Weitergabebeschränkungen nicht durch Verordnung aufgehoben werden können ( 5 Abs. 4).

Das Regelungswerk läßt erwarten, daß die Respondenten künftig spürbar entlastet werden. Der Gesetzgeber und noch mehr die für Verordnungen zuständigen Ministerien stehen unter Handlungszwang, die fehlenden Rechtsgrundlagen zu schaffen. Auch die grundsätzlich eingeräumte Möglichkeit, den Zugriff statt mit Gesetz durch Verordnung zu regeln, entlastet den Gesetzgeber von Routineentscheidungen und bringt schnellere Lösungen. Innerhalb des nationalen Statistiksystems ist ein enormer Fortschritt erzielt worden. 
Im Hinblick auf die europäischen Maßgaben besteht noch ein weiteres Entwicklungsund Anpassungspotential.

Es wäre durchaus im Sinne der Grundprinzipien (Prinzip Nr. 5), die Entscheidung über die jeweils sachgerechte Art der Erhebung dem Statistischen Amt zu überlassen. Dies wäre nicht nur ein Ausfluß der technical autonomy, sondern würde auch zu einer weiteren wesentlichen Entlastung der Verordnungsbürokratie führen.

Artikel 6 Abs. 1 Buchst. b) der Datenschutzrichtlinie wäre dadurch Rechnung getragen, daß das Verhältnis von Regel und Ausnahme angepaßt wird. Wenn der Datenzugang grundsätzlich mit dem ursprünglichen Verwaltungszweck kompatibel ist, dann sollten nur die Fälle in der die Statistik anordnenden Rechtsgrundlage geregelt werden, bei denen der Zugang ausnahmsweise nicht, oder nur beschränkt, erfolgen soll.

Die Verordnung über Gemeinschaftsstatistiken hat in Artikel 16 Abs. 1 das Recht auf Registerzugang konstituiert. Als unmittelbar geltendes Recht bedarf dessen Anwendbarkeit keiner nationalrechtlichen Bestätigung durch Gesetz oder Verordnung. Damit gehört bei Gemeinschaftsstatistiken die Frage, ob ein Zugang zu Verwaltungsdaten zulässig ist, auch nicht zu den noch erforderlichen Regelungen, die nach $\S 4$ Abs. 4 durch Verordnung festgelegt werden können oder müssen. Dem kann auch nicht mit Hinweis auf Artikel 16 Abs. 2 begegnet werden, denn die den Mitgliedstaaten verbleibende Kompetenz, die praktischen Vorkehrungen sowie die Einschränkungen und Voraussetzungen bei Bedarf regeln zu können, bezieht sich nicht das Zugangsrecht, sondern nur auf den tatsächlichen Zugang.

\section{Ergebniserstellung mit Daten, die auf verschiedenen Rechtsgrundlagen erhoben wurden}

Ganz ähnlich stellen sich die Fragen auch bezüglich der Zulässigkeit der Zusammenführung und Aufbereitung von Daten, die auf der Basis verschiedener Rechtsgrundlagen erhoben wurden, deren Informationen über statistische Einheiten in dem Rohmaterial sich aber ergänzen. Dieser Sachverhalt fehlt in der Auflistung des $\S 5$ Abs. 2, so daß eine derartige ergänzende Aufbereitung noch nicht einmal durch Verordnung geregelt werden könnte. Eine gleichartige Beschränkung der Nutzungsmöglichkeit vorhandener statistischer Daten findet sich in $\S 25$ Abs. 2, wonach nur die Registerdaten als solche aufbereitet werden dürfen, nicht aber als ergänzende Informationen im Zusammenhang mit anderen Statistiken. Für die Registerverwendung stellt sich darüber hinaus noch die Frage der Vereinbarkeit eines solchen Verbots mit der Verordnung (EWG) Nr. 2186/93 des Rates vom 22. Juli 1993 über die innergemeinschaftliche Koordinierung des Aufbaus von Unternehmensregistern für statistische Verwendungszwecke (Abl. Nr. L 196 vom 5.8.1993, S. 1). Statistische Zwecke, wie sie in der Überschrift und in Artikel 1 dieser Verordnung genannt sind, umfassen ohne weiteres die Verwendung für Aufbereitungen in einem über den Inhalt der Register hinausgehenden Zusammenhang. 
Adresse des Autors:

Christian Engelage

4, rue du Moulin

L-7328 Heisdorf

Luxembourg

Tel. und Fax: +352 332110

Email: Engelage@pt.lu 